\title{
Study on Self Repairing Method for Transient Fault and Low Energy Consumption of Digital Circuit
}

\author{
Huiling Si \\ Institute of Mechanical and Electrical Engineering, Zhengzhou University of Industrial Technology, \\ Zhengzhou 451100, China \\ xincao_123@126.com
}

Keywords: Digital circuit, transient fault, low energy consumption, self repairing.

\begin{abstract}
: at present, most of the digital circuit transient fault repair methods need to be completed by manual processing, the repair efficiency is low and the energy consumption is high. For this, put forward a new kind of digital circuit fault transient low energy self repair method, design a repair system, which is mainly composed of self reconfiguration control module and execution module, self-discipline of the core control module is embedded Nios processor, digital circuit in the presence of transient fault, the wavelet neural network algorithm the diagnosis of digital circuit transient fault judging circuit, whether the normal operation of. The reconfiguration execution module consists of a logical computing node LC and an internal interconnection network. The main function is to maintain the function of the digital circuit and carry out the information transmission between the other modules. The experimental results show that the proposed method can effectively diagnose the fault, the success rate and the average fitness of the fault repair are high, and the overall performance is strong.
\end{abstract}

\section{Introduction}

At present, digital circuits have been widely used in different products. In the process of using, there are some transient faults in digital circuits, which results in the failure of products to work properly [1]. Therefore, how to solve the transient failure of digital circuits with low energy consumption has become a great challenge for the relevant designers [2]. In order to make the product reliable and sustainable, it is necessary to use the automatic repair technology to deal with the transient faults of digital circuits [3].

A new low energy self repair method for transient failure of digital circuits is proposed in this paper. The experimental results show that the proposed method can effectively diagnose the fault, and the success rate and average adaptability of the fault repair are high, and the overall performance is strong. Design of Low - power Self - healing System for Digital Circuit Transient Faults.

\section{System design}

In order to realize the digital circuit fault transient low energy self repair, this section designs a repair system, which mainly consists of two parts, namely autonomic control module and the reconfiguration execution module, wherein the self-control module is the core of the system, its main role is a digital circuit transient fault detection, generating configuration information. At the same time, the information is transferred to the reconfiguration module for a specific logical calculation; The main function of the reconfiguration execution module is to maintain the function of the digital circuit and to carry out the information transfer between the modules and other modules. The overall framework of the system is shown in Fig. 1.

\subsection{Design of self - control unit}

Self-discipline control module is the core of embedded Nios II processors, digital circuits in the event of a transient fault cases, through the wavelet neural network algorithm for transient fault 
diagnosis of digital circuit, circuit judge whether the normal operation. This module can not only block the fault location, but also can successfully configure the information of specific functions [4]. The process of transient fault diagnosis of digital circuits is described below.

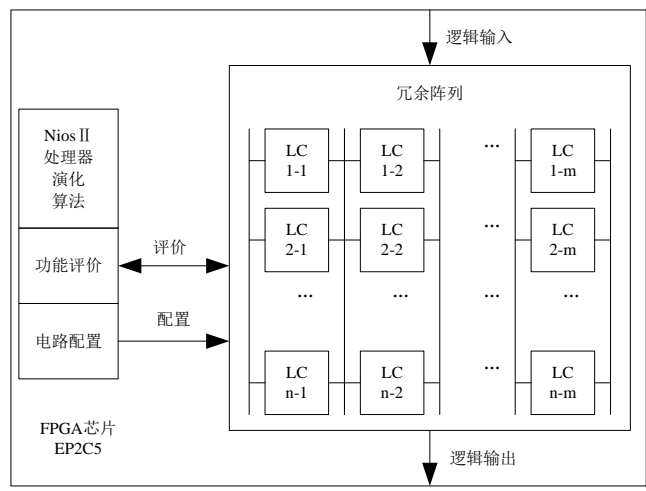

Fig. 1 System framework

\subsubsection{Feature extraction of transient fault in digital circuit}

In this section, the wavelet packet analysis method is used to extract the transient fault features of digital circuits. The basic principle of wavelet packet analysis is the localization of time frequency and frequency that can be changed by time and frequency. In the low frequency state, the frequency resolution is very high, the time resolution is very low, and it is in the opposite direction when it is in the high frequency state.

According to the characteristics of each alternative, the transient fault characteristics of digital circuits are extracted from different waveforms of different faults, and the final characteristic coefficients are obtained, which are regarded as transient fault feature vectors.

\subsubsection{Transient fault diagnosis of digital circuits}

According to the transient fault characteristics of the extracted digital circuits, we use neural network to diagnose transient faults. In this section, the transient fault feature vectors extracted from wavelet packets are used as inputs to neural network, and the dimension of transient fault feature vectors is described by $\mathrm{N}$. The number of nodes in input layer of neural network is also $\mathrm{N}$. Because the magnitude of the fault feature vectors is very different, the neural network can be output only after the normalization operation is completed.

When determining the output of the neural network, assuming that there are $\mathrm{M}$ different kinds of fault types in the digital circuit, the output of the neural network can be described as $\left\{y_{1}, y_{2}, \cdots, y_{i}, y_{M}\right\}$. If the digital circuit is in mode $i, y_{i}=0$, and the others, $\mathrm{y}_{i}=1$.The output of the neural network is $\{1,1, \cdots, 0,1\}$.

The three-layer neural network is used in this section. In the three layer neural network, the following relationship exists between the number of neurons in the hidden layer $n_{1}$, the number of neurons in the input layer $N$, and the number of output elements $M$.

$$
n_{1}=a \sqrt{N+M}
$$

In the form, a is used to describe the constant between $1 \sim 10$.

After the training of the neural network, the test sample is entered into the neural network, and the output results are obtained by learning, thus the type of the fault of the digital circuit is obtained.

\subsection{The design of reconfiguration execution module}

The system reconfiguration execution module includes the logical computing node LC and the internal interconnected network, and its structure diagram is described in Fig. 2. 


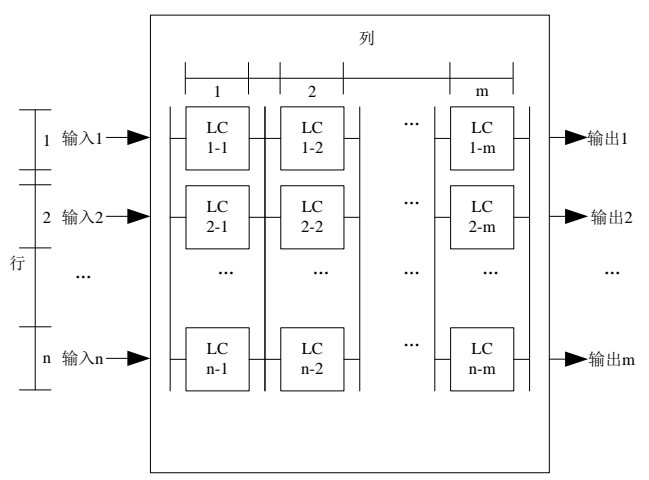

Fig. 2 Reconfiguration execution module structure

In Fig. 2, each calculation point corresponds to a computing function, and the data between these functions is managed through the internal internet. Therefore, a digital circuit composed of reconfigured execution modules can perform a specified logic operation.

\section{Analysis of experimental results}

\subsection{Test circuit and generation of transient fault}

In order to verify the effectiveness of the proposed method, it is necessary to carry out the relevant experimental analysis, and the digital circuit diagram of the experimental test is described in Fig. 3.

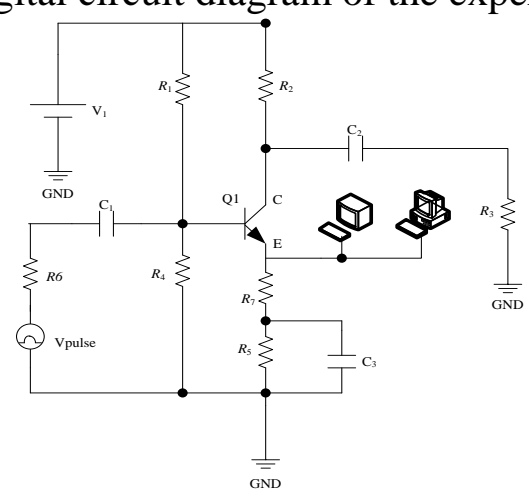

Fig. 3 Test circuit diagram

In the process of generating transient faults, the transient analysis of the circuit is carried out first, and the square wave signal is input. The voltage data of the one hundred time points of the $1 \mathrm{~ms} \sim 100 \mathrm{~ms}$ are selected as the test data.

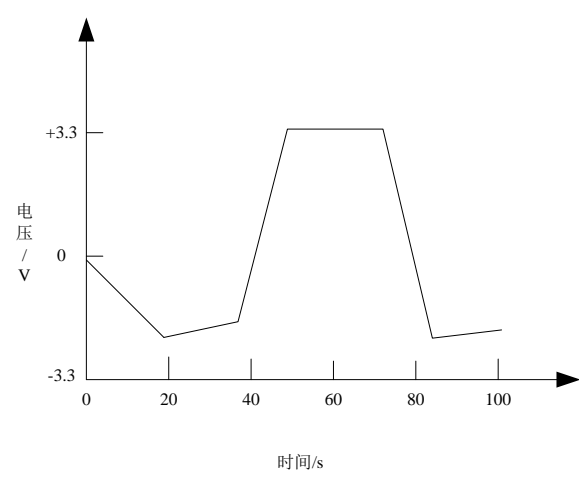

(a) Normal state

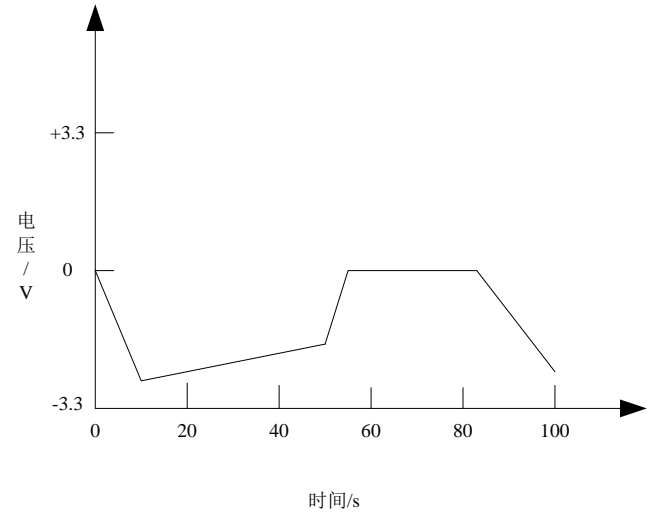

(b) The disconnected state of R1

Fig. 4 Circuit response curves under normal and transient faults 
In Fig. 4, the transient response curves of Normal state, the disconnected state of R1 are described respectively. The difference between normal state and transient fault state curve is quite different, so it can distinguish effectively.

\subsection{Test of transient fault diagnosis}

Transient fault diagnosis of digital circuits is the basis of transient fault repair for digital circuits. If fault diagnosis is wrong, the corresponding repair processing will have no meaning, resulting in reduced resource utilization and increased energy consumption.

This method is used to diagnose the transient short circuit fault of the digital circuit, and the instantaneous response curve of the output circuit is described in Fig. 5.

In Fig. 5 it can be seen that the fault diagnosis of digital circuit is carried out by this method. The output transient response curve is basically the same as the actual short circuit fault response curve, which shows that the diagnostic reliability of the method is high.

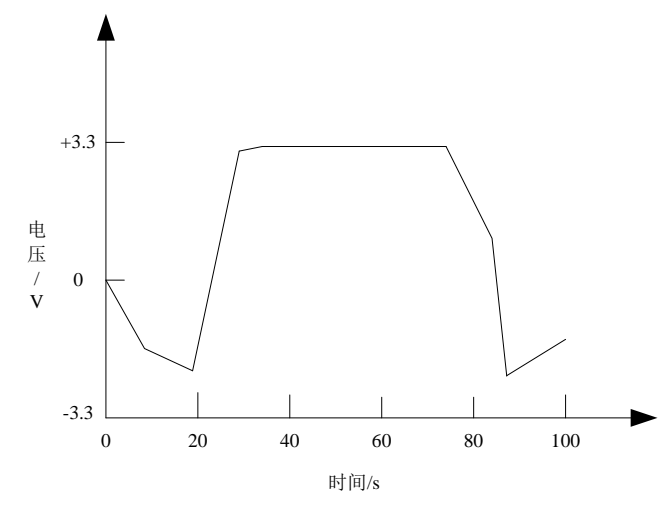

Fig. 5 Fault diagnosis results of this method

\section{Conclusions}

This paper presents a new digital circuit fault transient low energy self repair method, design a repair system, which is mainly composed of self reconfiguration control module and execution module, through the wavelet neural network algorithm for diagnosis of digital circuit transient fault judging circuit is not normal operation. The experimental results show that the proposed method can effectively diagnose the fault, and the success rate and average adaptability of the fault repair are high, and the overall performance is strong.

\section{References}

[1] Liang Huaguo, Yuan Deran, Yan Aibin, et al. Failure Probability Estimation for Digital Circuits Considering Single Event Multiple Transients[J]. Journal of Computer-Aided Design \& Computer Graphics, 2016,28(3):505-512.

[2] Liu Yijun, Zhang Li, Zhao Qiang, et al. Research of Low Energy Consumption Video Sensor Node Based on Distributed Video Coding[J]. Computer Measurement\&Control,2015,23(1):329-332.

[3] Gomes I A C, Martins M G A, Reis A I, et al. Exploring the use of approximate TMR to mask transient faults in logic with low area overhead[J]. Microelectronics Reliability, 2015, 55 (9-10): 2072-2076.

[4] Li Z, Han Y, Xu P. Methods for benchmarking building energy consumption against its past or intended performance: An overview[J]. Applied Energy, 2014, 124(7):325-334. 